\title{
DESENVOLVIMENTO DE APLICATIVO MÓVEL PARA CHECKLIST DE CIRURGIA SEGURA
}

\section{ARTIGO ORIGINAL}

SILVA, Rafael Henrique ${ }^{1}$

GATTI, Marcia Aparecida Nuevo ${ }^{2}$

MARTA, Sara Nader ${ }^{3}$

CARAM, Nirave Reigota ${ }^{4}$

FRANZOLIN, Solange de Oliveira Braga ${ }^{5}$

${ }^{1}$ Doutorado em Biologia Oral, Mestrado em Saúde Coletiva, Pós-graduação em Urgência e Emergência e em Centro Cirúrgico e Central de Material e Esterilização, Graduação em Enfermagem.

2 Doutorado em Doenças Tropicais pela Universidade Estadual Paulista Júlio de Mesquita Filho - UNESP/Botucatu, Mestrado em Saúde Coletiva pela Universidade Estadual Paulista Júlio de Mesquita Filho - UNESP/Botucatu. Pós-graduação em Administração dos Serviços de Saúde, Enfermagem do Trabalho e Metodologia do Ensino. Graduação em Enfermagem.

${ }^{3}$ Doutorado e Mestrado em Odontopediatria. Especialista em Odontopediatria e em Pacientes com Necessidades Especiais. Graduação em Odontologia.

${ }^{4}$ Doutorado em Educação Escolar, Mestrado em Televisão Digital (atual Mídia e Tecnologia), MBA em Marketing. Licenciatura em Marketing, Graduação em Pedagogia pela Universidade do Sagrado Coração (2019), Graduação em Tecnologia em Marketing, Graduação em Comunicação Social.

5 Doutorado em Fisiopatologia em Clínica Médica, Mestrado em Odontologia, Mestrado em Biotecnologia Médica, Graduação em Odontologia. 
PEDRO, Ketilin Mayra ${ }^{6}$

LOPES, Jaqueline de Souza ${ }^{7}$

SILVA, Rafael Henrique. Et al. Desenvolvimento de aplicativo móvel para checklist de cirurgia segura. Revista Científica Multidisciplinar Núcleo do Conhecimento. Ano 05, Ed. 08, Vol. 10, pp. 25-38. Agosto de 2020. ISSN: 2448-0959, Link de acesso: https://www.nucleodoconhecimento.com.br/saude/checklist-de-cirurgia

\section{RESUMO}

As tecnologias de comunicação e informação estão influenciando de forma crescente as ações na área da saúde, assim como a segurança do paciente. Desta forma, este trabalho teve como objetivo desenvolver um aplicativo para conferência e controle de todas as etapas do checklist de Cirurgia Segura sugerido pela Organização Mundial da Saúde melhorando a segurança do paciente submetido à cirurgia. O problema da pesquisa está justamente na ausência de aplicativos para dispositivos móveis capaz de atender a necessidade de segurança do paciente. Trata-se de estudo aplicado no desenvolvimento tecnológico de um aplicativo com possibilidade de implantação em qualquer serviço de saúde e de fácil instalação em dispositivos móveis. O aplicativo foi construído baseado no checklist de Cirurgia Segura estabelecido pela Organização Mundial da Saúde. O aplicativo permite a identificação do paciente através de três identificadores. Posteriormente contempla todos os itens das três etapas do checklist de cirurgia segura: Antes da Indução Anestésica, Antes da Incisão Cirúrgica e Antes do Paciente Sair da Sala. Ao final de todos as etapas do aplicativo, ele calcula o risco para a segurança do paciente. $O$ aplicativo desenvolvido é uma ferramenta que pode

6 Pós-doutorado pela Universidade Estadual Paulista. Doutorado e Mestrado em Educação. Graduação em Pedagogia.

7 Pós-graduação em Saúde Indígena pelo Programa de Residência Multiprofissional da Universidade Federal da Grande Dourados. Graduação em Enfermagem. 
ser implantada nas instituições de saúde e utilizada pelos profissionais que atuam no centro cirúrgico.

Palavras-chave: Segurança do paciente, procedimentos cirúrgicos operatórios, smartphone.

\section{INTRODUÇÃO}

A sociedade contemporânea é constituída por indivíduos, empresas e Estado operando num campo local, nacional e internacional numa estrutura definida como Sociedade em Rede. O funcionamento dessa nova estrutura social é dependente de tecnologias digitais de comunicação e informação (CASTELLS; CARDOSO, 2005). Após a inovação causada pela internet, a popularização dos celulares inteligentes (smatfones) é considerada a revolução tecnológica mais impactante dos últimos anos (TIBES et al., 2014; IDC, 2013).

A popularização dos smartphones mudou a relação com a tecnologia, visto que esses aparelhos desempenham a função de computador de bolso, uma vez que permite uma variedade de possibilidade devido a sua grande diversidade de aplicativos. Essa funcionalidade similar ao computador acontece graças ao desenvolvimento dos Sistemas Operacionais avançados para smartphones (SOUZA, 2016).

Bilotti et al. (2017) afirma que atualmente, o smartphone permite o acesso fácil à internet com um custo relativamente baixo. Ele permite acesso à aplicativos que abordam os mais variados temas, inclusive pode ser uma alternativa para melhorar o acesso da população à saúde, bem como a promoção e prevenção.

A área da saúde certamente é uma das mais influenciadas pelo crescente uso das tecnologias de comunicação e informação. Com o passar dos anos, a utilização de dispositivos móveis cresceu e ganhou cada vez mais espaço nessa área, dando origem ao conceito de mobile health (mHealth) definido como práticas médicas e de saúde pública auxiliadas por aparatos portáteis, como celulares, aparelhos de 
monitoramento dos pacientes, assistentes pessoais digitais e outros aparelhos sem fio (WHO, 2011).

Os aplicativos, em sua grande maioria, são criados por desenvolvedores particulares e não estão vinculados a projetos de pesquisa, desta forma, apesar da popularização dos aplicativos e da sua importância para a área da saúde, a pesquisa nesse meio ainda é incipiente (TIBES et al., 2014). Muitas vezes os aplicativos da área da saúde são idealizados por profissionais e concretizados por concretizados por profissionais de tecnologia da informação, no entanto, sem necessariamente passarem por uma metodologia adequada e vinculadas a projetos de pesquisa (OLIVEIRA; ALENCAR, 2017).

Segundo Souza et al. (2013) houve nos últimos anos um aumento no número de profissionais da saúde que utilizam de smartphones em suas atividades laborais. Esse fato ilustra uma nova realidade na prestação de serviços, onde os aplicativos por meio de dispositivos móveis, facilitam a consulta de guidelines, protocolos e acompanhamento de pacientes.

Entre os protocolos atuais, ganha destaque mundial os voltados para a segurança do paciente cirúrgico, com orientação para que seja instituída como uma política de saúde que deve ser implementada e monitorada com rigor em todos os serviços de saúde, tanto públicos quanto privados. Para o paciente que necessita realizar uma cirurgia é fundamental que os procedimentos anestésicos e cirúrgicos ocorram com qualidade e que a cultura de segurança do paciente possibilite que os possíveis erros sejam minimizados através da aplicação do Protocolo para Cirurgia Segura da Organização Mundial da Saúde (OMS) (CORONA; PENICHE, 2015).

A OMS utilizou de três princípios para desenvolver o Checklist, simplicidade, ampla aplicabilidade e possibilidade de mensuração do impacto. Ele permite que as equipes, ao seguirem de forma eficiente as etapas críticas de segurança, possam minimizar os riscos evitáveis mais comuns, que colocam em risco as vidas e comprometem o bemestar dos pacientes cirúrgicos (ELIAS et al., 2015). 
O Checklist é composto por 19 itens divididos em três momentos distintos: antes da indução anestésica, antes da incisão cirúrgica e antes de o paciente sair da sala de operações (OLIVEIRA et al., 2018; AMAYA et al., 2015; PAIVA et al., 2015; SORIAALEDO et al., 2012).

Antes da Indução Anestésica: É confirmado verbalmente com o paciente a identidade do mesmo, o procedimento e o local da cirurgia, e se o consentimento para o procedimento foi assinado. Confere se o lado correto da cirurgia foi sinalizado e o funcionamento adequado do oxímetro de pulso. A equipe de anestesia deve rever verbalmente se o paciente possui vias aéreas de difícil acesso, risco de perda sanguínea ou de reação alérgica (OMS, 2009).

Antes de Iniciar a Cirurgia: Os profissionais se apresentam com nome e função. Em voz alta, é conferido a identidade do paciente, o procedimento e local do corpo a ser operado. Posteriormente, o cirurgião, o anestesista e a equipe de enfermagem revisam verbalmente os pontos críticos para a cirurgia. Em seguida, é confirmado o uso profilático de antibióticos nos últimos 60 minutos e a disponibilidade dos exames de imagem (OMS, 2009).

Antes do Paciente Sair da Sala Cirúrgica: Antes do paciente ser encaminhado à sala de recuperação anestésica, a equipe analisa o procedimento realizado, confere a quantidade de compressas e instrumentais cirúrgicos, identifica as peças anatômicas, checa possíveis danos nos equipamentos e problemas a serem resolvidos e traça planos de cuidados em relação ao pós-operatório do paciente (OMS, 2009).

$\mathrm{Na}$ área da saúde a utilização de checklist é uma prática relativamente recente, no entanto, em áreas como aviação, setor de energia nuclear e construção civil já faz parte da rotina do processo de trabalho, pois permite a checagem de itens importantes que poderiam ser esquecidos pela equipe. Essas áreas utilizam de tecnologia na estruturação e implantação desse processo, prática essa que poderia ser incorporada para segurança do paciente (FREITAS et al., 2014). 
Desta forma, o objetivo do trabalho foi desenvolver um aplicativo para conferência e controle de todas as etapas do checklist de Cirurgia Segura sugerido pela Organização Mundial da Saúde melhorando a segurança do paciente submetido à cirurgia.

\section{MÉTODOS}

Trata-se de estudo aplicado no desenvolvimento tecnológico de um aplicativo com possibilidade de implantação em qualquer serviço de saúde e de fácil instalação em dispositivos móveis.

O desenvolvimento do aplicativo envolveu uma proposta construtivista e consistiu na ação intencional de planejar, desenvolver e aplicar situações específicas, incorporando mecanismos que favoreçam a contextualização.

O aplicativo foi construído baseado no checklist de Cirurgia Segura estabelecido pela Organização Mundial da Saúde. A OMS permite que cada instituição adapte o Checklist de Cirurgia Segura para a sua realidade, no entanto, como o intuito é que o aplicativo possa ser utilizado nas mais diversas instituições e realidades, optou-se por utilizar o Checklist padrão preconizado pela OMS.

O desenvolvimento ocorreu através do trabalho do próprio autor com uma equipe de programação. Esse momento compreendeu a definição de como as etapas do Checklist seriam apresentadas no aplicativo, a definição da estrutura de navegação, as ferramentas do aplicativo e o planejamento da configuração de ambientes.

O aplicativo móvel está disponível em um site com link para download em smartphone ou tablet com sistema operacional Android. Posteriormente ao seu desenvolvimento, o aplicativo foi testado durante procedimentos cirúrgicos em um Hospital de Ensino.

O estudo foi realizado após aprovação do Comitê de Ética em Pesquisa em Seres Humanos da Universidade Sagrado Coração - Bauru/SP. 


\section{RESULTADOS}

A apresentação inicial do aplicativo foi desenvolvido de forma que o profissional faça a inserção dos dados referente ao procedimento que será realizado.

Figura 1. Tela inicial do aplicativo

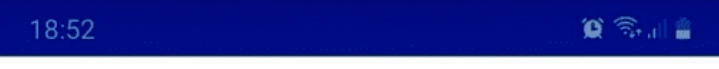

Checklist de Cirurgia Segura

Nome do Paciente

\begin{tabular}{l}
\hline Data de nascimento \\
\hline Nome da mãe \\
\hline Nome do cirurgião \\
\hline Nome do procedimento \\
AVANÇAR
\end{tabular}

18:53 四 ำ จำ

\section{Checklist de Cirurgia Segura}

\begin{tabular}{l} 
Teste1 \\
\hline $01 / 01 / 2000$ \\
\hline Teste2 \\
\hline Teste3 \\
\hline Teste4 \\
\hline
\end{tabular}

Fonte: Próprio autor.

Posteriormente, as questões do checklist de Cirurgia Segura da OMS serão apresentadas para que cada etapa seja realizada durante o procedimento e posteriormente respondida. Caso a etapa for corretamente realizada e o item preenchido, o aplicativo irá automaticamente para a etapa seguinte, como apresentado a seguir: 
Figura 2. Tela para identificação do paciente, local e procedimento.

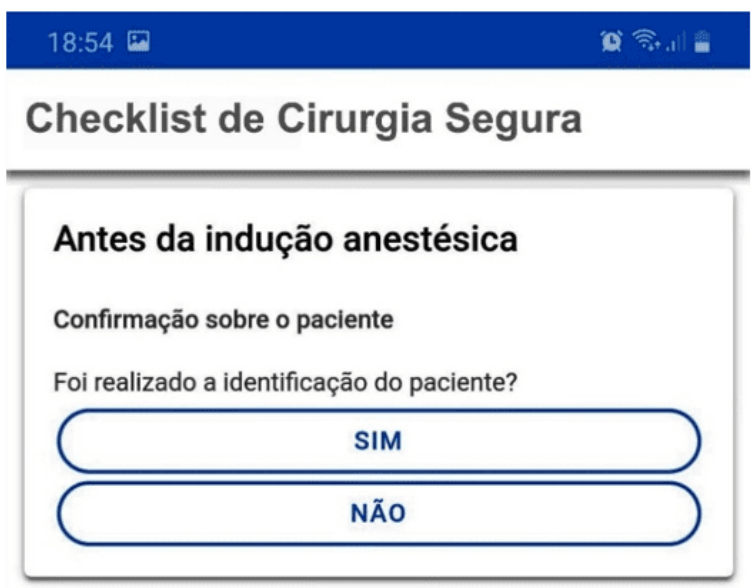

18:54 田 के

\section{Checklist de Cirurgia Segura}

\section{Antes da indução anestésica}

Confirmação sobre o paciente

Foi confirmado o local da cirurgia e o procedimento?

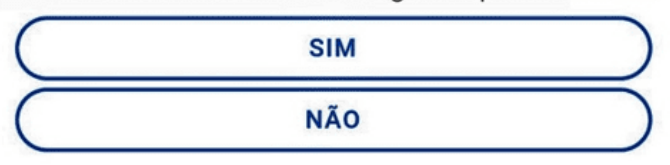

Fonte: Próprio autor.

No caso da etapa Antes da indução anestésica, no item confirmação sobre o paciente, aplicativo questiona se foi confirmado o local da cirurgia e o procedimento a ser feito, caso o profissional não responda conforme o orientado pela OMS, o aplicativo irá trazer um alerta, informando que essa atitude acarretará em um risco para o paciente e questiona se o profissional deseja continuar. Caso o profissional responda que deseja continuar, o aplicativo passará para o próximo item. Se o profissional informar que não deseja continuar, o aplicativo retorna para a tela anterior solicitando a confirmação do local da cirurgia e o procedimento a ser realizado.

Enquanto o profissional não realizar a etapa corretamente ou não informar que pretende ir para a próxima etapa, mesmo estando ciente do risco para a segurança do paciente, o aplicativo permanecerá questionando o profissional. 
Figura 3. Questionamento do aplicativo frente a ação que representa um risco para o paciente

Checklist de Cirurgia Segura
Antes da indução anestésica
Confirmação sobre o paciente
Essa ação representa um risco ao paciente. Deseja
NÃo
SIM

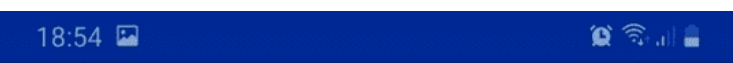

\section{Checklist de Cirurgia Segura}

\section{Antes da indução anestésica}

Confirmação sobre o paciente

Foi confirmado o local da cirurgia e o procedimento?

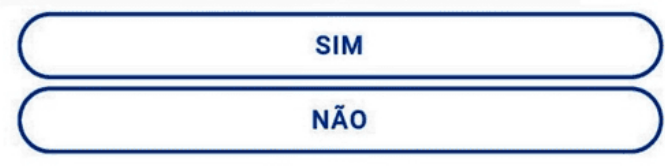

Fonte: Próprio autor.

$\mathrm{Na}$ sequência, o aplicativo continua com os questionamentos da etapa antes da indução anestésica. $\mathrm{Na}$ tela seguinte, o aplicativo questiona o profissional sobre o termo de consentimento cirúrgico, em seguida sobre o sítio cirúrgico demarcado, posteriormente sobre a checagem do equipamento cirúrgico e sobre o oxímetro de pulso instalado e funcionando.

$\mathrm{Na}$ tela referente ao item onde o profissional deve questionar o paciente sobre alguma alergia, caso a resposta seja negativa, o aplicativo seguirá para a etapa seguinte. Porém, caso o paciente informe que possui algum tipo de alergia, o profissional irá responder que sim e o aplicativo abrirá um campo para que seja informado qual é a alergia do paciente, seja alguma medicação, material ou alimento.

Finalizando os itens que devem ser realizados antes da indução anestésica, o aplicativo irá questionar quanto ao risco de via aérea difícil e o risco de perda sanguínea.

Na sequência das etapas do Checklist de Cirurgia Segura, os questionamentos são referentes a etapa "Antes de iniciar a cirurgia". Da mesma forma, caso a resposta seja a adequada $o$ aplicativo segue para $o$ item seguinte, caso a resposta seja inadequada, 
o aplicativo alerta o profissional sobre o risco para a segurança do paciente e se, ainda assim, deseja continuar.

Nessa etapa, também conhecida como time out ou pausa cirúrgica, o aplicativo atua auxiliando os profissionais sobre todos os questionamentos necessários. Uma tela do aplicativo questiona se todos os profissionais da equipe confirmam seus nomes e profissões, a tela seguinte solicita que o cirurgião, anestesista e enfermeiro confirmem a identidade do paciente, o local e o procedimentos a ser realizado. Posteriormente, cirurgião, anestesista e enfermagem são indagadas pelo aplicativo se há informações relevantes que necessitam ser compartilhadas. Por fim, o aplicativo de cirurgia segura questiona sobre a antibioticoprofilaxia nos últimos 60 minutos antes da incisão e a disponibilidade dos exames de imagem na sala de cirurgia.

A terceira etapa do Checklist de Cirurgia Segura é "Antes do paciente sair da sala de cirurgia". O aplicativo também contempla todos os itens dessa etapa, em uma tela solicita a confirmação do procedimento realizado, na seguinte a contagem de compressas, instrumentos e agulhas, na próxima tela se as biópsias estão identificadas, posteriormente o aplicativo questiona o profissional se houve algum problema com os equipamentos utilizados, e na tela seguinte, se cirurgião, anestesista e enfermeiro analisam os pontos mais importantes na recuperação pós-anestésica e pós-operatória do paciente.

Ao final do processo, o aplicativo apresenta um cálculo da porcentagem das etapas realizadas corretamente em conformidade com as etapas do Check-list: Antes da indução anestésica, Antes da incisão cirúrgica e Antes do paciente sair da sala de cirurgia. Com as porcentagens calculadas, o aplicativo realiza um último cálculo e informa o risco do paciente durante o procedimento de acordo com o Check-list de Cirurgia Segura preconizado pela OMS. 
Figura 4. Cálculo do risco do paciente gerado pelo aplicativo

$$
\text { 18:56 国 }
$$

\section{우.}

\section{Checklist de Cirurgia Segura}

\section{Antes da indução anestésica}

$\mathbf{8 3} \%$ dos itens realizados

\section{Antes de iniciar a cirurgia}

$\mathbf{8 6} \%$ dos itens realizados

\section{Antes do paciente sair da sala cirúrgica}

$\mathbf{8 0} \%$ dos itens realizados

\section{Geral}

De acordo com o Check-list de Cirurgia Segura da OMS o risco desse paciente durante $\circ$ procedimento é de $17 \%$

\section{RESUMO}

\section{INícıO}

Fonte: Próprio autor. 
O aplicativo apresentará as opções Resumo e Início. Na opção INÍCIO o aplicativo retorna para a primeira tela para iniciar novamente o Check-list. O profissional pode escolher a opção RESUMO onde o aplicativo gera um relatório com todas as questões do Check-list e as respectivas respostas.

Há ainda a opção imprimir que permite imprimir o relatório em uma impressora configurada ou salvar o arquivo em PDF no Tablet ou Smartphone.

\section{DISCUSSÃO}

O aplicativo de cirurgia segura foi desenvolvido considerando que os aplicativos da área da saúde voltados para cirurgia segura serviam apenas de consulta às etapas do checklist da OMS. A funcionalidade desse aplicativo permite a interação do profissional com todas as etapas do checklist da OMS, promovendo a segurança do paciente e estimulando os profissionais a seguirem corretamente todas as etapas.

Além de melhorar a comunicação na sala operatória, o checklist é uma ferramenta para garantir segurança ao paciente. Há uma dificuldade de verbalizar informações por parte de alguns profissionais, desta forma o checklist oportuniza que os profissionais compartilhem eventuais preocupações com os demais membros da equipe (PANCIERI et al., 2014; CUNAT et al., 2011; HAYNES et al., 2011; PAUGAMBURTZ, GUERRERO, 2011; RATEAU et al., 2011).

Em relação ao paciente, optou-se por três identificações: nome, data de nascimento e nome da mãe. Mais de uma identificação favorece a segurança do paciente e os identificadores escolhidos possibilitam ao paciente responde-los com maior facilidade. Em seguida é necessário preencher o nome do cirurgião e o procedimento que será realizado.

A identificação correta do paciente é válida e necessária para que toda a equipe cirúrgica não execute procedimentos errôneos. (ALPENDRE et al., 2017; FREITAS et al., 2014). Essa confirmação garante que a cirurgia seja realizada no paciente correto. Identificar o paciente é fundamental para garantir que a assistência seja prestada ao 
paciente correto, considerando a possibilidade de troca de paciente. Diante desta possibilidade, a identificação do paciente, segundo protocolo, é preconizado pelo Ministério de Saúde para todas as instituições que prestam assistência à saúde. (AMAYA et al., 2015; MINISTÉRIO DA SAÚDE, 2013).

O aplicativo é uma ferramenta digital, o seu comprometimento é com a segurança do paciente, no entanto, o tempo utilizado para responder todas as etapas do aplicativo não deveria onerar o tempo de procedimento, desta forma o aplicativo foi elaborado para agilizar o checklist, com isso, em média, o tempo utilizado em todas as etapas do aplicativo foi de 144 segundos, ou seja, 2 minutos e 24 segundos.

Estima-se que o tempo necessário para aplicação das três etapas do checklist é de três minutos. A orientação é que seu preenchimento seja fácil, rápido e que um único profissional seja responsável por conduzir sua aplicação. (SOUZA et al., 2016; PANCIERI et al., 2013).

A informatização do processo, através de aplicativos de dispositivos móveis, permite que as informações sejam registradas em um menor tempo e reduzindo a possibilidade de registros equivocados. (LEE et al., 2018).

O aplicativo desenvolvido calculou que um único item não realizado aumenta o risco do paciente em $7 \%$, desta forma, espera-se que o profissional ao visualizar essa informação possa rever a sua conduta.

Resultados de estudos nacionais e internacionais expõe que as inconformidades no procedimento de cirurgia segura não ocorrem exclusivamente por limitações de recursos ou infraestrutura dos centros cirúrgicos, precisando de uma abordagem muito mais complexa. Identificar os fatores relacionados à adesão ao checklist $\mathrm{e}$ avaliar os métodos de implementação efetivos são ações essenciais para melhor compreensão do fenômeno e avançar no processo de segurança do paciente. (ALMEIDA, RODRIGUES, 2018; CULLATI et al., 2013; PICKERING et al., 2013).

Um estudo observou que o uso do checklist foi realizado em $80 \%$ das cirurgias, no entanto, o registo de itens não confirmados evidenciou problemas relacionados à 
fidedignidade das informações registradas. É preciso destacar que o preenchimento sem a verificação incorre em aspectos éticos e legais para todos os profissionais da equipe cirúrgica. Essa prática gera uma importante reflexão sore a segurança do paciente, visto que ao não serem verificados, cada item coloca o paciente em risco de sofrer eventos adversos. (MAZIERO et al., 2015; SORIA-ALEDO et al., 2012).

A OMS orienta que as instituições podem acrescentar itens ao checklist para que ele possa atender a sua realidade. No entanto, nessa adequação, a orientação é que os itens sejam apenas incluídos conforme a realidade das instituições e nunca excluir itens já preconizados e com evidência científica favorável. (RIBEIRO et al., 2017; HAYNES et al., 2009).

No desenvolvimento do aplicativo optou-se por manter os itens de verificação exatamente como o checklist de cirurgia segura do protocolo da OMS. Essa decisão foi tomada para que o aplicativo possa ser utilizado em todas as instituições de saúde e atenda a necessidade geral de promoção de segurança do paciente. Posteriormente, se necessário, instituições ou profissionais podem solicitar que itens sejam acrescentados para atender as suas particularidades.

A OMS alerta que acrescentar muitos itens pode tornar o checklist demasiadamente complexo, dificultando sua incorporação ao processo de trabalho e à cultura organizacional. (RIBEIRO et al., 2017; FREITAS et al., 2014; WHO, 2011).

O aplicativo foi elaborado da forma simples e intuitiva, focando na agilidade e pensando na segurança do paciente. Todos os profissionais que vierem a utilizar o aplicativo devem ser instruídos como ele funciona e sobre a importância para a segurança do paciente e dos profissionais envolvidos na cirurgia.

A implantação de um novo instrumento precisa passar por um processo de capacitação de toda equipe e provocar percepções individuais entre os membros da equipe cirúrgica explicando a importância de cada item do checklist. (ELIAS et al., 2015; CARNEY et al., 2012; CONLEY et al., 2011). 
Implantar o protocolo de cirurgia segura é um desafio a ser enfrentado pelos profissionais e instituições. Uma assistência cirúrgica com segurança é mais do que apenas realizar as etapas do procedimento anestésico-cirúrgico e aplicar uma lista de verificação. Uma mudança na cultura de segurança do paciente é necessária e os gestores das instituições de saúde e os profissionais da equipe cirúrgica precisam compreendam os desafios e os benefícios do protocolo. (SOUZA et al., 2016; CORONA; PENICHE, 2015).

\section{CONCLUSÃO}

O aplicativo desenvolvido é uma ferramenta que pode ser implantada nas instituições de saúde e utilizada pelos profissionais que atuam no centro cirúrgico. Após os testes realizados em procedimentos cirúrgicos constatou-se que ele atende as necessidades de segurança do paciente. $\mathrm{O}$ aplicativo permite a interação com todas as etapas do checklist de cirurgia segura e calcula automaticamente o risco para o paciente a cada etapa não realizada. Espera-se que a utilização do aplicativo promova ações voltadas para a segurança do paciente e que difunda entre as instituições e profissionais o protocolo de cirurgia segura.

\section{REFERÊNCIAS}

ALMEIDA, R. E.; RODRIGUES, M. C. S. Preenchimento da Lista de Verificação de Segurança Cirúrgica em hospitais brasileiros. Rev. Rene. 2018.

ALPENDRE, F. T. et al. Ciclo PDCA para elaboração de checklist de segurança cirúrgica. Cogitare Enferm. (22) 3: e50964, 2017.

AMAYA, M. R. et al. Análise do registro e conteúdo de checklists para cirurgia segura. Esc. Anna Nery, 19 (2): 246-251, 2015.

BILOTTI, C. C. et al. M-Health no controle do câncer de colo do útero: pré-requisitos para o desenvolvimento de um aplicativo para smartphones. Rev. Eletron. Comum. Inf. Inov. Saúde, abr.-jun.; 11(2), 2017. 
CARNEY, B. T. et al. Differences in nurse and surgeon perceptions of teamwork: implications for use of a briefing checklist in the OR. AORN J. 91(6):722-9, 2012.

CASTELLS, M.; CARDOSO, G. A Sociedade em Rede do Conhecimento à Ação Política. Imprensa Nacional. Casa da Moeda, 2005.

CONLEY, D. M. et al. Effective surgical safety checklist implementation. J Am Coll Surg., 212(5):873-9, 2011.

CORONA, A. R. P. D.; PENICHE, A. C. G. A Cultura de Segurança do Paciente na Adesão ao Protocolo de Cirurgia Segura. Rev. SOBECC, São Paulo. Jul./Set.; 20(3): 179-185, 2015.

CULLATI, S. et al. Is the Surgical Safety Checklist successfully conducted? An observational study of social interactions in the operating rooms of a tertiary hospital. BMJ Qual Saf., 22(8):639-46, 2013.

CUNAT, C. et al. Stratégie de deployment de la checklist dans un CHU. Ann Fr Anesth Reanim. 30(6):484-8, 2011.

ELIAS, A. C. G. P. et al. Avaliação da adesão ao checklist de cirurgia segura. Rev. SOBECC, São Paulo. Jul./Set. 20(3): 128-133, 2015.

FREITAS, M. R. et al. Avaliação da adesão ao checklist de cirurgia segura da OMS em cirurgias urológicas e ginecológicas, em dois hospitais de ensino de Natal, Rio Grande do Norte, Brasil. Cad. Saúde Pública, 2014.

HAYNES, A. B. et al. Changes in safety attitude and relationship to decreased postoperative morbidity and mortality following implementation of a checklist-based surgical safety intervention. BMJ Qual Saf. 20:102-7, 2011.

IDC (INTERNATIONAL DATA CORPORATION). Worldwide Business Use Smartphone 2013-2017 Forecast and Analysis. Framingham, 2013. 
LEE, K. et al. Effect of self-monitoring on long-term patient engagement with mobile health applications. PLoS ONE, 13(7), 2018.

MAZIERO, E. C. S. et al. Adesão ao uso de um checklist cirúrgico para segurança do paciente. Rev Gaúcha Enferm. dez; 36(4): 14-20, 2015.

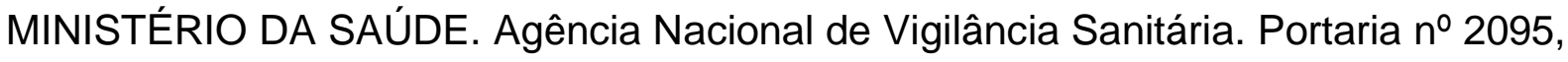
de 24 de setembro de 2013. Aprova os protocolos básicos de segurança do paciente. Brasília: Ministério da Saúde, 2013.

OLIVEIRA, A. R.; ALENCAR, M. S. M. O uso de aplicativos de saúde para dispositivos móveis como fontes de informação e educação em saúde. Rev. Digit. Bibliotecon. Cienc. Inf. Campinas, SP. v.15 n.1 p.234-245, 2015.

OLIVEIRA, M. C. B. et al. Adesão do cheklist cirúrgico à luz da cultura de segurança do paciente. Rev. SOBECC, São Paulo. jan./mar. 23(1): 36-42, 2018.

OMS. Segundo desafio global para a segurança do paciente: Cirurgias seguras salvam vidas. Brasília (DF): Agência Nacional de Vigilância Sanitária; 2009.

PAIVA, A. C. R. et al. Checklist de cirurgia segura: análise do preenchimento da ficha de verificação no pré, trans e pós-operatório. Rev. Enfermagem Revista, v. 18, n. 02. mai/ago, 2015.

PANCIERI, A. P. et al. Aplicação do checklist para cirurgia segura: Relato de experiência. Rev. SOBECC, São Paulo. jan/mar. 19(1): 26-33, 2014.

PANCIERI, A. P. et al. Safe surgery checklist: analysis of the safety and communication of teams from a teaching hospital. Rev. Gaúcha Enferm. 34(1): 71-8, 2013.

PAUGAM-BURTZ, C.; GUERRERO, O. Check-list sécurité au bloc opératoire: le bilan après un an de deployment à l'hôpital Beaujon. Ann Fr Anesth Reanim. 30(6): 4758, 2011. 
PICKERING, S. P. et al. Compliance and use of the World Health Organization checklist in UK operating theatres. Br J Surg. 100(12): 1664-70, 2013.

RATEAU, F. et al. Check-list "Patient Safety" in the operating room: one year experience of 40,000 surgical procedures at the university hospital of Nice. Ann Fr Anesth Reanim.30:479-83, 2011.

RIBEIRO, H. C. T. C. et al. Adesão ao preenchimento do checklist de segurança cirúrgica. Cad. Saúde Pública, 33(10), 2017.

SORIA-ALEDO, V. et al. Dificultades en la implantación del checklist en los quirófanos de cirugía. Cir Esp, 90:180-5, 2012.

SOUZA, C. M. Aplicativos para smartphones e sua colaboração na capacidade funcional de idosos. Rev. Saúde Digi. Tec. Edu. Fortaleza; v.1, n.1, p.06-19, 2016.

SOUZA, R. C. et al. Processo de criação de um aplicativo móvel na área de odontologia para pacientes com necessidades especiais. Revista da ABENO, 13(2): 58-61, 2013.

SOUZA, R. M. et al. Aplicabilidade do checklist de cirurgia segura em centros cirúrgicos hospitalares. Rev. Sobecc, São Paulo. out./dez; 21(4): 192-197, 2016.

TIBES, C. M. S. et al. J. Aplicativos móveis desenvolvidos para a área da saúde no Brasil: revisão integrativa da literatura. Rev. Min. Enferm. 18(2): 471-478, 2014.

WHO (WORLD HEALTH ORGANIZATION). Global Observatory for eHealth. mHealth, New Horizons for Health trough Mobile Technologies. Geneva (Switzerland): WHO; 2011.

Enviado: Julho, 2020.

Aprovado: Agosto, 2020. 